\title{
Bilateral recurrent laryngeal nerve palsy in a patient undergoing consecutive thyroid operations
}

\author{
In-Suk Kwak ${ }^{1}$, Taewan $\mathrm{Lim}^{2}$, Yul Oh${ }^{2}$, Young Ri Kim², and Yoon Kyung Lee ${ }^{1}$ \\ Department of Anesthesiology and Pain Medicine, ${ }^{1}$ Hangang Sacread-Heart Hospital, Seoul, ${ }^{2}$ Dongtan Sacread-Heart Hospital, \\ Hwasung, Korea
}

A 57-year-old woman $(162 \mathrm{~cm}, 65 \mathrm{~kg})$ was admitted with breast pain. An ultrasound scan revealed an $8 \mathrm{~mm}$ nodule in the right thyroid gland. The nodule was confirmed as a thyroid carcinoma with fine needle aspiration biopsy and thyroidectomy was performed. In the preoperative testing, vocal cord (VC) motion was normal on both sides despite suspected laryngeal reflux. No other specific clinical features were found before the surgery. Because of a loose front tooth, the thyroidectomy was performed with a $7 \mathrm{~mm}$ internal diameter endotracheal tube (ETT) via a light wand. No breathing difficulties were present at extubation, and no postoperative airway symptoms were found. In a $\mathrm{VC}$ test performed 2 days after the surgery, voice changes were detected, and a fiber-optic bronchoscope (FOB) examination exhibited incomplete paralysis of the right VC. Histopathologic examination of the postoperative specimen showed a positive end margin, so a left thyroidectomy with extensive neck dissection was scheduled three days after the right thyroidectomy.

No medications were administered preoperatively. She had normal sinus rhythm, oxygen saturation of $98 \%$ without oxygen, blood pressure of $135 / 80 \mathrm{mmHg}$ and pulse of 50 . She did not have specific airway symptoms except for voice changes before the anesthesia. Anesthesia was induced with $40 \mathrm{mg}$ lidocaine, $120 \mathrm{mg}$ propofol, $40 \mathrm{mg}$ rocuronium and 8 vol\% sevoflurane. Because of a loose front tooth, intubation with a $7 \mathrm{~mm}$ internal diameter ETT was performed using a light wand. The balloon of the ETT was confirmed to be over the suprasternal notch, and normal breath sounds were checked and found. The ETT was fixed at a distance of $20 \mathrm{~cm}$ from the corner of the mouth. Cuff pressure was maintained as low as possible to prevent leakage. Anesthesia was maintained with $\mathrm{FiO}_{2}$ of 0.5 , oxygen of $0.9 \mathrm{~L} / \mathrm{min}$, air of $1.6 \mathrm{~L} / \mathrm{min}$ and sevoflurane of 3-4 vol\%.

The surgery took 65 minutes to perform and was without problems, but the surgeon suggested that there was a possibility of damage to the left recurrent laryngeal nerve (RLN) from the LigaSure $^{\mathrm{TM}}$ sutures. Pyridostigmine $(15 \mathrm{mg})$ and glycopyrrolate (0.6 mg) were administered for recovery. She regained consciousness with normal breathing within 10 minutes with the ETT still in place. Because of possible damage to the left RLN and potential breathing difficulties that might be caused by incomplete paralysis of the right VC, extubation was performed in the operating room in preparation for reintubation and so that tracheostomy could be performed instantly in an emergency. The patient claimed she had difficulty breathing after the extubation. Mask support was applied for breathing assistance and $10 \mathrm{mg}$ of dexamethasone was administered. FOB was performed within 15 minutes after the extubation.

Both the VCs were immobile, and the space between the two vocal folds was less than $5 \mathrm{~mm}$, meaning that the airway was not completely blocked. Breathing difficulties continued and oxygen saturation stayed at around $90 \%$ despite oxygen delivery. Because of the need for breathing assistance, tracheostomy or reintubation was considered. However, a normal range of oxygen saturation returned as a result of the steady breathing assistance given. The patient was moved to the intensive care unit (ICU) 55 minutes after the extubation.

A FOB performed in the ICU revealed reduced motion of the arytenoid cartilage and a $5 \mathrm{~mm}$ space between the two vocal

Corresponding author: Taewan Lim, M.D., Department of Anesthesiology and Pain Medicine, Hallym University Dongtan Sacred Heart Hospital, 40, Seoku-dong, Hwaseong 445-907, Korea. Tel: 82-31-8086-2283, Fax: 82-31-8086-2029, E-mail: 1twhanzo@naver.com (c) This is an open-access article distributed under the terms of the Creative Commons Attribution Non-Commercial License (http:// creativecommons.org/licenses/by-nc/3.0/), which permits unrestricted non-commercial use, distribution, and reproduction in any medium, provided the original work is properly cited. 


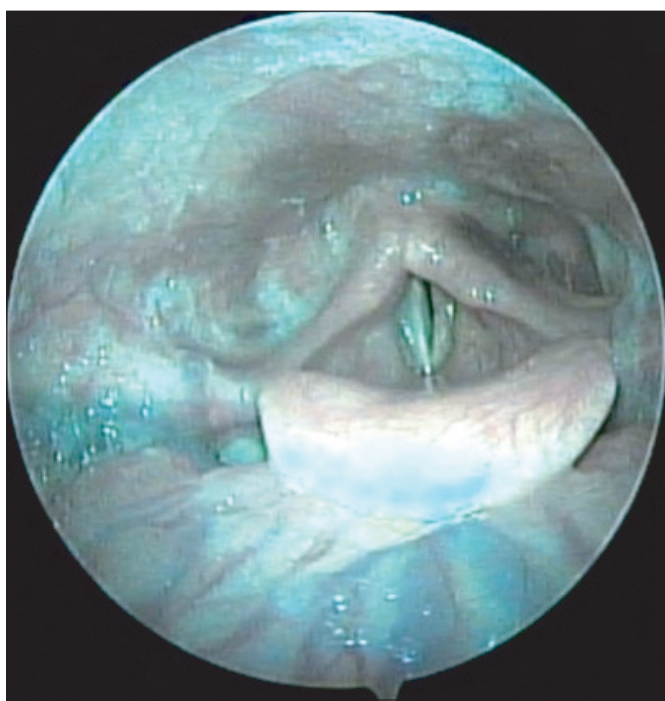

Fig. 1. Fiber-optic bronchoscope.

folds (Fig. 1), so the oxygen saturation was continually monitored while oxygen was given via an oxygen mask. She began to have less breathing difficulties although her voice remained hoarse. As she had no breathing problems at 20 hours postoperatively, she was transferred to the open ward and discharged seven days after the surgery. During the outpatient follow-up, left VC lateral fixation was performed 24 and 26 days postoperatively under the diagnosis of bilateral VC paralysis. In the endoscopic examination performed 3 months postoperatively, the left VC was retracted laterally after the lateral fixation. The right VC had fully recovered its function.

The incidence of RLN damage following thyroid surgery is not frequent. However, it can cause catastrophic obstruction when both VCs become paralyzed [1]. Unilateral VC paralysis does not pose a threat to life, but it can be critical when accompanied by breathing difficulties or diaphragmatic paralysis. The case described herein can be also serious because VC paralysis on the one side was subsequently followed by VC paralysis on the other side. Symptomless unilateral RLN palsy prediction is difficult to evaluate pre-operatively without VC evaluation [2]. Moreover, an overlooked postoperative voice change can cause airway management problems in the situation of bilateral VC palsy as described herein. RLN damage can be examined during surgery as follows: the VCs can be observed through the laryngeal mask using a FOB [3], RLN can be stimulated using a stimulator [4], and an electromyogram can be performed with an endotracheal tube containing an electrode (reinforced EMG probe, Medtronic Xomed, Jacksonville, Florida, USA) [5]. In this case report, breathing difficulties can be treated in a timely manner when bilateral VC palsy is identified, in a step before extubation. During the preparation for reintubation, tracheostomy and supportive ventilation are required for extubation, and so an operation room is a suitable place to perform an extubation. Then a VC test should be performed promptly using an FOB. In this case report, we did not perform reintubation because the oxygen saturation increased with supportive ventilation. Besides, reintubation would have made a VC test impossible. The laryngeal mask was not used because she had a loose front tooth and risked having airway spasm. Considering the complications of tracheostomy, it should be the last option but it should be considered when the patient presents with cardiovascular disease, obesity or a neuropsychiatric disorder [1]. Bilateral VC palsy after surgery usually improves over time with supportive care. Therefore, securing an emergent airway should not be performed impetuously. RLN damage can heal on its own in several months, but otherwise a laryngeal operation such as lateral fixation is necessary as evidenced in this case report [1].

When thyroid surgery is performed in a patient with unilateral VC palsy on the opposite side from the affected thyroid, the possibility of bilateral VC palsy should be considered. The medical history should be checked and a VC test is necessary when there is any symptom. Detection of the presence or absence of laryngeal nerve damage should be checked during surgery, and preparation for possible breathing problems is essential even when there is no laryngeal nerve damage on the opposite side.

\section{References}

1. Zábrodský M, Bouček J, Kastner J, Kuchař M, Chovanec M, Betka J. Immediate revision in patients with bilateral recurrent laryngeal nerve palsy after thyroid and parathyroid surgery. How worthy is it? Acta Otorhinolaryngol Ital 2012; 32: 222-8.

2. Weiss A, Isselhorst C, Gahlen J, Freudenberg S, Roth H, Hammerschmitt N, et al. Acute respiratory failure after deep cervical plexus block for carotid endarterectomy as a result of bilateral recurrent laryngeal nerve paralysis. Acta Anaesthesiol Scand 2005; 49: 715-9.

3. Shah EF, Allen JG, Greatorex RA. Use of the laryngeal mask airway in thyroid and parathyroid surgery as an aid to the identification and preservation of the recurrent laryngeal nerves. Ann R Coll Surg Engl 2001; 83: 315-8.

4. Alon EE, Hinni ML. Transcricothyroid electromyographic monitoring of the recurrent laryngeal nerve. Laryngoscope 2009; 119: 1918-21.

5. Julien N, Mosnier I, Bozorg Grayeli A, Nys P, Ferrary E, Sterkers O. Intraoperative laryngeal nerve monitoring during thyroidectomy and parathyroidectomy: a prospective study. Eur Ann Otorhinolaryngol Head Neck Dis 2012; 129: 69-76. 